\title{
Ultrafast Spin Dynamics in Optically Excited Bulk GaAs at Low Temperatures
}

\author{
M. Krauß,${ }^{1}$ R. Bratschitsch, ${ }^{2}, *$ Z. Chen,${ }^{2}$ S. T. Cundiff, ${ }^{2}$ and H. C. Schneider ${ }^{1, \dagger}$ \\ ${ }^{1}$ Physics Department and Research Center OPTIMAS, \\ University of Kaiserslautern, P. O. Box 3049, 67663 Kaiserslautern, Germany \\ ${ }^{2}$ JILA, National Institute of Standards and Technology and University of Colorado, Boulder, CO 80390-0440, USA
}

(Dated: October 31, 2018)

\begin{abstract}
This paper presents a study of electron spin dynamics in bulk GaAs at low temperatures for elevated optical excitation conditions. Our time-resolved Faraday rotation measurements yield subnanosecond electron spin dephasing times over a wide range of $n$-doping concentrations in quantitative agreement with a microscopic treatment of electron spin dynamics. The calculation shows the occurrence and breakdown of motional narrowing for spin dephasing under elevated excitation conditions. We also find a peak of the spin dephasing time around a doping density for which, under lower excitation conditions, a metal-insulator transition occurs. However, the experimental results for high excitation can be explained without a metal-insulator transition. We therefore attribute the peak in spin-dephasing times to the influence of screening and scattering on the spin-dynamics of the excited electrons.

PACS numbers: 72.25.Rb,71.70.Ej,78.47.-p,72.25.Fe
\end{abstract}

\section{INTRODUCTION}

Electron spin dynamics in semiconductors has experienced a dramatic revival of interest in the last decade due to the promise of semiconductor-based spintronics applications. ${ }^{1,2}$ In contrast to magneto-electronic systems involving metallic multilayers, semiconductors are better suited for the fabrication of integrated electronic and opto-electronic devices, but in non-ferromagnetic semiconductors the spin is not a conserved quantity due to the spin-orbit interaction. Thus an understanding of spin relaxation in semiconductors is essential, because it limits all information processing and storage using electronic spins. The renewed interest in combination with improved experimental techniques has led to important new results. For instance, long spin dephasing times in n-doped GaAs at low temperatures and low carrier densities have been measured using time-resolved Faraday rotation (FR) techniques. ${ }^{3,4}$ Very recently, the technique of spin noise measurements has provided a new tool for the determination of "intrinsic" spin relaxation times without electrical or optical carrier injection. ${ }^{5}$ These measurements show, for instance, the importance of a metal-insulator transition on the spin relaxation at low temperatures. However, the foundation for the theoretical explanation of these experiments above the transition temperature was already laid by an analysis of Dyakonov and Perel (DP $)^{6}$ who showed that in n-doped GaAs the decay of spin polarization occurs through the dephasing of the spin coherence in a spatially inhomogeneous effective magnetic field provided by the band structure.

Although these "non-invasive" experimental techniques yield important results, ${ }^{7}$ one may also be interested in the excitation of appreciable spin-polarized carrier densities by optical or electronic means. Existing studies, which address these questions, have concentrated on the electron spin dynamics in n-doped GaAs-based semiconductors under electrical bias and Hanle effect measurements on a timescale of several hundred picoseconds. ${ }^{8}$

This paper is concerned with an investigation of the spin-dephasing dynamics in n-doped bulk GaAs after the excitation of spin-polarized carrier densities by ultrashort optical pulses. For a wide range of doping densities, we present a detailed comparison between time-resolved FR measurements and a microscopic theory, which is based on the original DP analysis. Our approach to compute the spin-dephasing time does not require the determination of auxiliary quantities, such as an effective momentum scattering time, which is often employed to achieve agreement with experiment. ${ }^{9}$ Instead, the equations of motion for the spin and momentum-resolved electronic distribution functions are solved numerically including the relevant effects of the band-structure and the scattering of electrons with other electrons, phonons, and impurities. ${ }^{10}$ Due to the numerical complexity of calculating the spin dynamics in bulk GaAs, theoretical results so far have concentrated on the microscopic spin dynamics in low-dimensional semiconductors, ${ }^{11,12}$ with the exception of recent work, ${ }^{13}$ or on accurate calculations of the analytical DP results for the spin decay. ${ }^{14,15}$

This paper is organized as follows. In Sec. II we explain the theoretical approach and present the dynamical equations for the spin-density matrix. In Sec. III we describe the experimental setup and our modeling of the experimental conditions in the framework of the theory. Sec. IV contains the experimental results and their interpretation by comparing them with model calculations. 


\section{THEORETICAL APPROACH}

We directly calculate the dynamics of the momentum resolved $2 \times 2$ spin-density matrix

$$
\rho_{s s^{\prime}}(\boldsymbol{k})=\left\langle c_{s, \boldsymbol{k}}^{\dagger} c_{s^{\prime} \boldsymbol{k}}\right\rangle
$$

where $s=\uparrow, \downarrow$ denotes the spin projection quantum number along the quantization axis $z, c\left(c^{\dagger}\right)$ are electron destruction (creation) operators in the Heisenberg picture, and $\langle\cdots\rangle$ denotes the average with respect to the equilibrium statistical operator. The elements of the spin-density matrix are the carrier occupation numbers $n_{\boldsymbol{k}}^{s}=\rho_{s s}(\boldsymbol{k})$ and the complex coherences $\Psi_{\boldsymbol{k}}=\rho_{\uparrow \downarrow}(\boldsymbol{k})$. In terms of the spin density-matrix, the average spin is given by

$$
\begin{aligned}
\left\langle s_{x}(\boldsymbol{k})\right\rangle & =\frac{\hbar}{2} \operatorname{Re}\left[\Psi_{\boldsymbol{k}}\right] \\
\left\langle s_{y}(\boldsymbol{k})\right\rangle & =\frac{\hbar}{2} \operatorname{Im}\left[\Psi_{\boldsymbol{k}}\right] \\
\left\langle s_{z}(\boldsymbol{k})\right\rangle & =\frac{\hbar}{2}\left(n_{\boldsymbol{k}}^{\uparrow}-n_{\boldsymbol{k}}^{\downarrow}\right) .
\end{aligned}
$$

The time evolution of $\rho(\boldsymbol{k})$ is determined by the equation of motion

$$
\frac{\partial \rho(\boldsymbol{k})}{\partial t}=\left.\frac{\partial \rho(\boldsymbol{k})}{\partial t}\right|_{\mathrm{coh}}+\left.\frac{\partial \rho(\boldsymbol{k})}{\partial t}\right|_{\text {scatt }}
$$

where the coherent part includes the electronic energies, the influence of the effective magnetic field due to the band structure, and Hartree-Fock renormalizations. For the diagonal elements $n_{\boldsymbol{k}}^{s}$ we have

$$
\begin{aligned}
\left.\frac{\partial}{\partial t} n_{\boldsymbol{k}}^{s}\right|_{\text {coh }}= & -\frac{2 s}{\hbar}\left\{\left[g \mu_{B} B+\Omega_{x}(\boldsymbol{k})\right] \operatorname{Im}\left(\Psi_{\boldsymbol{k}}\right)+\Omega_{y}(\boldsymbol{k}) \operatorname{Re}\left(\Psi_{\boldsymbol{k}}\right)\right\} \\
& +\frac{4 s}{\hbar} \operatorname{Im} \sum_{\boldsymbol{q}} W_{\boldsymbol{q}} \Psi_{\boldsymbol{k}+\boldsymbol{q}}^{*} \Psi_{\boldsymbol{k}}
\end{aligned}
$$

andthe off-diagonal elements are given by

$$
\begin{aligned}
\left.\frac{\partial}{\partial t} \Psi_{\boldsymbol{k}}\right|_{\text {coh }}= & \frac{1}{2 \hbar}\left[i g \mu_{B} B+i \Omega_{x}(\boldsymbol{k})+\Omega_{y}(\boldsymbol{k})\right]\left(n_{\boldsymbol{k}}^{\uparrow}-n_{\boldsymbol{k}}^{\downarrow}\right)-\frac{i}{\hbar} \Omega_{z}(\boldsymbol{k}) \Psi_{\boldsymbol{k}} \\
& +\frac{i}{\hbar} \sum_{\boldsymbol{q}} W_{\boldsymbol{q}}\left[\left(n_{\boldsymbol{k}+\boldsymbol{q}}^{\uparrow}-n_{\boldsymbol{k}+\boldsymbol{q}}^{\downarrow}\right) \Psi_{\boldsymbol{k}}-\Psi_{\boldsymbol{k}+\boldsymbol{q}}\left(n_{\boldsymbol{k}}^{\uparrow}-n_{\boldsymbol{k}}^{\downarrow}\right)\right] .
\end{aligned}
$$

Here, $s= \pm 1 / 2$ denotes the electron spin and $\boldsymbol{B}=B \hat{e}_{x}$ in the Voigt geometry. The screened Coulomb interaction

$$
W_{\boldsymbol{q}}=\frac{1}{\tilde{\varepsilon}_{\mathrm{bg}} \tilde{\varepsilon}_{\boldsymbol{q}}} v_{q}
$$

in Eqs. (6) and (7) contains the bare Coulomb potential

$$
v_{\boldsymbol{q}}=\frac{e^{2}}{\varepsilon_{0}} \frac{1}{q^{2}}
$$

and the dimensionless dielectric functions $\tilde{\varepsilon}_{\mathrm{bg}}$ and $\tilde{\varepsilon}_{\boldsymbol{q}}$. The former describes the "background" screening due to the polarizability of the lattice, and the latter the screening due to the electrons provided by the doping. For this, we use the static limit of the Lindhard dielectric function ${ }^{16}$ in the numerical evaluation. The scattering contributions (electron-electron, electron-phonon, and electron-impurity) to Eq. (5) can be obtained by equation-of-motion or Green's function techniques ${ }^{17-19}$ following, e.g., Refs. 20-22. As an example we write out the expressions for Coulomb scattering and define the kernels for in- and out-scattering

$$
K_{\boldsymbol{k}_{1}, \boldsymbol{q}}^{\mathrm{in}}=\left(1-n_{\boldsymbol{k}_{1}+\boldsymbol{q}}^{\uparrow}\right) n_{\boldsymbol{k}_{1}}^{\uparrow}+\left(1-n_{\boldsymbol{k}_{1}+\boldsymbol{q}}^{\downarrow}\right) n_{\boldsymbol{k}_{1}}^{\downarrow}-2 \operatorname{Re}\left(\Psi_{\boldsymbol{k}_{1}+\boldsymbol{q}}^{*} \Psi_{\boldsymbol{k}_{1}}\right)
$$

and

$$
K_{\boldsymbol{k}_{1}, \boldsymbol{q}}^{\mathrm{out}}=n_{\boldsymbol{k}_{1}+\boldsymbol{q}}^{\uparrow}\left(1-n_{\boldsymbol{k}_{1}}^{\uparrow}\right)+n_{\boldsymbol{k}_{1}+\boldsymbol{q}}^{\downarrow}\left(1-n_{\boldsymbol{k}_{1}}^{\downarrow}\right)-2 \operatorname{Re}\left(\Psi_{\boldsymbol{k}_{1}+\boldsymbol{q}}^{*} \Psi_{\boldsymbol{k}_{1}}\right)
$$


so that the scattering contribution for the diagonal elements becomes

$$
\begin{aligned}
\left.\frac{\partial}{\partial t} n_{\boldsymbol{k}}^{s}\right|_{\text {scat }}= & \frac{2 \pi}{\hbar} \sum_{\boldsymbol{k}_{1}, \boldsymbol{q}} W_{\boldsymbol{q}}^{2} \delta\left(\epsilon_{\boldsymbol{k}}-\epsilon_{|\boldsymbol{k}+\boldsymbol{q}|}+\epsilon_{\left|\boldsymbol{k}_{1}+\boldsymbol{q}\right|}-\epsilon_{\boldsymbol{k}_{1}}\right) \\
& \times\left(K_{\boldsymbol{k}_{1}, \boldsymbol{q}}^{\mathrm{in}}\left[\left(1-n_{\boldsymbol{k}}^{s}\right) n_{\boldsymbol{k}+\boldsymbol{q}}^{s}-\operatorname{Re}\left(\Psi_{\boldsymbol{k}}^{*} \Psi_{\boldsymbol{k}+\boldsymbol{q}}\right)\right]\right. \\
& \left.-K_{\boldsymbol{k}_{1}, \boldsymbol{q}}^{\text {out }}\left[n_{\boldsymbol{k}}^{s}\left(1-n_{\boldsymbol{k}+\boldsymbol{q}}^{s}\right)-\operatorname{Re}\left(\Psi_{\boldsymbol{k}}^{*} \Psi_{\boldsymbol{k}+\boldsymbol{q}}\right)\right]\right) .
\end{aligned}
$$

For the off-diagonal elements we have

$$
\begin{aligned}
\left.\frac{\partial}{\partial t} \Psi_{\boldsymbol{k}}\right|_{\text {scat }}= & \frac{\pi}{\hbar} \sum_{\boldsymbol{k}_{1}, \boldsymbol{q}} W_{\boldsymbol{q}}^{2} \delta\left(\epsilon_{\boldsymbol{k}}-\epsilon_{|\boldsymbol{k}+\boldsymbol{q}|}+\epsilon_{\left|\boldsymbol{k}_{1}+\boldsymbol{q}\right|}-\epsilon_{\boldsymbol{k}_{1}}\right) \\
& \times\left(K_{\boldsymbol{k}_{1}, \boldsymbol{q}}^{\mathrm{in}}\left[\Psi_{\boldsymbol{k}+\boldsymbol{q}}\left(2-n_{\boldsymbol{k}}^{\uparrow}+n_{\boldsymbol{k}}^{\downarrow}\right)-\Psi_{\boldsymbol{k}}\left(n_{\boldsymbol{k}+\boldsymbol{q}}^{\uparrow}+n_{\boldsymbol{k}+\boldsymbol{q}}^{\downarrow}\right)\right]\right. \\
& \left.-K_{\boldsymbol{k}_{1}, \boldsymbol{q}}^{\mathrm{out}}\left[\Psi_{\boldsymbol{k}}\left(2-n_{\boldsymbol{k}+\boldsymbol{q}}^{\uparrow}+n_{\boldsymbol{k}+\boldsymbol{q}}^{\downarrow}\right)-\Psi_{\boldsymbol{k}+\boldsymbol{q}}\left(n_{\boldsymbol{k}}^{\uparrow}+n_{\boldsymbol{k}}^{\downarrow}\right)\right]\right) .
\end{aligned}
$$

In Eqs. (12) and (13), the electronic energy dispersions are given by $\epsilon_{k} \equiv \epsilon_{k}^{\mathrm{e}}=\hbar^{2} /(2 m) k^{2}$ with the electron effective mass $m_{\mathrm{e}}=0.067 m_{0}$. An important step for the numerical solution of Eq. (5) for bulk GaAs is to account for the strongly anisotropic intrinsic magnetic field $\boldsymbol{\Omega}(\boldsymbol{k})$ by an expansion of $\rho(\boldsymbol{k})$ in a set of orthonormal functions $L_{\ell}(\vartheta, \varphi)$. Such an expansion was already used in Ref. 23 but, in contrast to the original treatment, we choose an expansion in functions that describe the angular dependence of $\boldsymbol{\Omega}$ most accurately, namely: $L_{1}(\vartheta, \varphi) \propto 1$ and $L_{2,3,4}(\vartheta, \varphi) \propto \Omega_{x, y, z}$ where the components of the intrinsic magnetic field are given by

$$
\begin{aligned}
& \Omega_{x}(\boldsymbol{k})=\gamma k_{x}\left(k_{y}^{2}-k_{z}^{2}\right) \\
& \Omega_{y}(\boldsymbol{k})=\gamma k_{y}\left(k_{z}^{2}-k_{x}^{2}\right) \\
& \Omega_{z}(\boldsymbol{k})=\gamma k_{z}\left(k_{x}^{2}-k_{y}^{2}\right)
\end{aligned}
$$

with a material specific constant $\gamma \cdot{ }^{24}$

Before we turn to the numerical results from this theory, which describes nonequilibrium spin dynamics due to the Dyakonov-Perel mechanism, it should be noted that there exists a variety of effects which lead to relaxation and dephasing of spin polarizations in GaAs. However, in moderately n-doped GaAs at low temperatures under elevated optical excitation conditions, the Dyakonov-Perel mechanism is dominant. The quantitative agreement between the parameter free microscopic calculation and the Faraday rotation measurements additionally, presented in Sec. IV, supports this point of view. For definiteness, we mention the following competing processes: Besides the intrinsic magnetic field $\boldsymbol{\Omega}$, the $g$-factor dispersion of electrons in $\mathrm{GaAs}^{2}$ leads to additional dephasing. We neglect this effect because the optically excited spin polarization is due to electrons in a small energy interval above the Fermi energy (less than $10 \mathrm{meV}$ ). In this region the spread of rotation frequencies due to the $g$-factor dispersion $\Delta g \mu_{\mathrm{B}}|\boldsymbol{B}|$ is typically one order of magnitude smaller than the internal magnetic fields $\hbar\langle|\boldsymbol{\Omega}(\boldsymbol{k})|\rangle$. Thus the dephasing due to the $g$-factor dispersion is of minor importance for the theoretical analysis of the experiments presented in this paper. The BirAronov-Pikus mechanisms usually acts on a longer timescale for the relatively low density of optically excited holes and therefore does not play a dominant role, either. ${ }^{25,26}$ Relaxation due to interactions with a nuclear spin polarization is not included in the calculation; in the experimental results it is excluded by periodically switching the helicity of the circularly polarized excitation beam.

\section{EXPERIMENT}

We have performed ultrafast FR measurements to determine the electron spin dynamics in n-doped bulk GaAs layers. ${ }^{1,27,28}$ The doped GaAs wafer was mounted on a sapphire substrate and thinned down to about $30 \mu \mathrm{m}$ for the transmission measurement. Spin polarized electrons were optically excited by a circularly polarized pump pulse. In this setup, the ultrafast laser pulses are generated by a mode-locked Ti:sapphire laser oscillator operating at $76 \mathrm{MHz}$ repetition frequency. The samples were held at low temperatures in an optical cryostat with a split-coil superconducting magnet, and a magnetic field of typically $4 \mathrm{~T}$ was applied. After the excitation, the electron spins begin to precess in a magnetic field applied parallel to the sample surface (Voigt geometry). By measuring the polarization rotation of a linearly polarized probe beam of the same wavelength transmitted through the sample, the electron spin dynamics can be monitored directly. The spin precession results in an oscillating signal, as shown in Fig. 1, that decays exponentially. We use an exponential fit for the signal envelope to extract the decay time due to dephasing processes, which is conventionally denoted ${ }^{2}$ by the ensemble spin dephasing time $T_{2}^{*}$. 


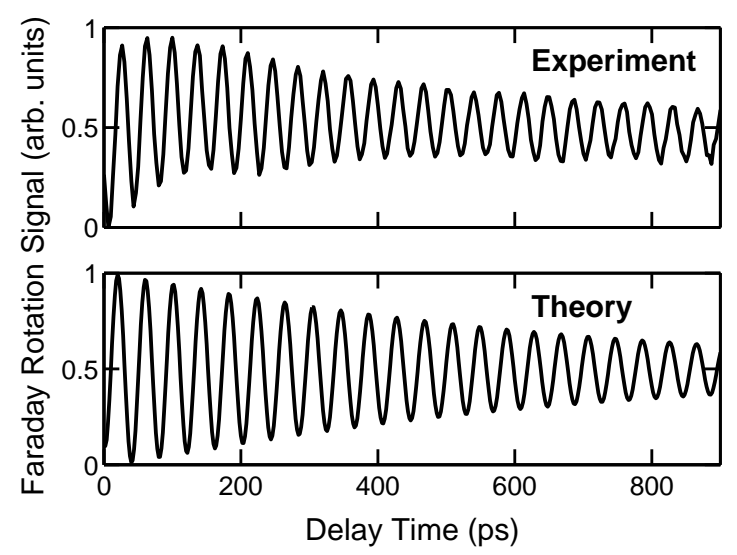

FIG. 1: Time-resolved Faraday rotation signal for $N_{\text {dop }}=3.6 \times 10^{16} \mathrm{~cm}^{-3}, B=4 \mathrm{~T}$, and $T=4 \mathrm{~K}$ as obtained by experiment (top), and numerical calculation (bottom). For the calculation $N_{\text {exc }}=1 \times 10^{16} \mathrm{~cm}^{-3}$ was assumed. The spin-dephasing time $T_{2}^{*}$ is determined by the exponential decay time of the envelope.

For the comparison with the experiment, we use for the calculation initial carrier distributions $n_{k}^{0}=n_{k}^{\text {dop }}+n_{k}^{\text {exc }}$, which contain both the influence of the doping and the optical excitation, because the excitation pulse duration is much shorter than the timescale of the subsequent electronic spin dynamics, so that any coherences introduced by the pulse can be ignored for the electronic dynamics. The itinerant carriers introduced by the doping are assumed to be completely relaxed, and are modeld by unpolarized Fermi-Dirac electron distributions $n^{\text {dop }}=f\left(\epsilon_{k}^{\mathrm{e}}\right)$ at lattice temperature with electronic density equal to the density of dopants. We model the electronic carrier distribution $n_{k}^{\text {exc }}$ excited by the ultrashort circularly polarized laser pulse propagating in $z$ direction as a Gaussian with spectral width of the pulse, $\sigma=15 \mathrm{meV}$. In accordance with the experimental procedure, we take the photon energy $\hbar \omega_{\mathrm{p}}$ approximately equal to the electron-hole transition energy at the Fermi momentum of the doping density under consideration. As in the experiment, we include a magnetic field of $4 \mathrm{~T}$, a lattice temperature $4 \mathrm{~K}$. Further we choose a $50 \%$ initial spin polarization of the optically excited carriers, which corresponds to the optimal spin polarization achievable by optical orientation at the bandgap. ${ }^{23}$ For the calculation of the time-resolved FR signal shown in Fig. 1, we assume a linearly polarized probe pulse degenerate with the circularly polarized pump. Assuming that the holes are unpolarized ${ }^{29,30}$ on the timescale of interest and that excitonic effects do not contribute, the FR signal is determined by ${ }^{31}$

$$
\Theta_{\mathrm{F}} \propto \sum_{\boldsymbol{k}} g(\boldsymbol{k})\left[n_{\boldsymbol{k}}^{\uparrow}-n_{\boldsymbol{k}}^{\downarrow}\right]
$$

Here, $g(\boldsymbol{k}) \propto \exp \left[\left(\epsilon_{k}^{\mathrm{e}}-\epsilon_{k}^{\mathrm{h}}-\hbar \omega_{\mathrm{p}}\right)^{2} / \sigma^{2}\right]$, with $\sigma$ the spectral width of the pulse and $\epsilon_{k}^{\mathrm{h}}=\hbar^{2} k^{2} /\left(2 m_{\mathrm{h}}\right), m_{\mathrm{h}}=0.457 m_{0}$, contains the contribution of the electron-hole transition at wavevector $\boldsymbol{k}$ to the signal at the probe photon energy $\hbar \omega_{\mathrm{p}}$. For the experimental and theoretical "raw data," as shown, for instance, in Fig. 1, we use the same exponential fit for the decay of the signal envelope to extract $T_{2}^{*}$.

\section{RESULTS}

Figure 2 shows the dependence of the spin-dephasing time $T_{2}^{*}$ on the photoexcited carrier density. Both in theory and experiment we find monotonically decreasing dephasing times for n-doped GaAs $\left(N_{\mathrm{dop}}=3.6 \times 10^{16} \mathrm{~cm}^{-3}\right)$ and increasing dephasing times for undoped GaAs with the better agreement for the undoped GaAs. When comparing measured and calculated results, it should be noted that there is a considerable uncertainty in the experiment because the photoexcited carrier density is difficult to estimate, and for the doped sample, we have an additional uncertainty in the doping density. Taking this into account, the agreement for the doped GaAs is very good. The agreement for undoped GaAs is even better because the weak dependence of the spin-dephasing time on the excited carrier density means that the experimental uncertainty in the optically excited carrier density has only a limited influence.

The spin-dephasing times for undoped and n-doped GaAs in Fig. 2 differ by more than a factor of three and have different slopes due to the different spin dynamics in both cases: In the undoped material, the spin of a nonequilibrium, highly spin-polarized (50\%) electron plasma relaxes, whereas in the doped material the spin dynamics of polarized optically excited electrons occurs in the presence of unpolarized electrons residing in the material due to the n-doping. 


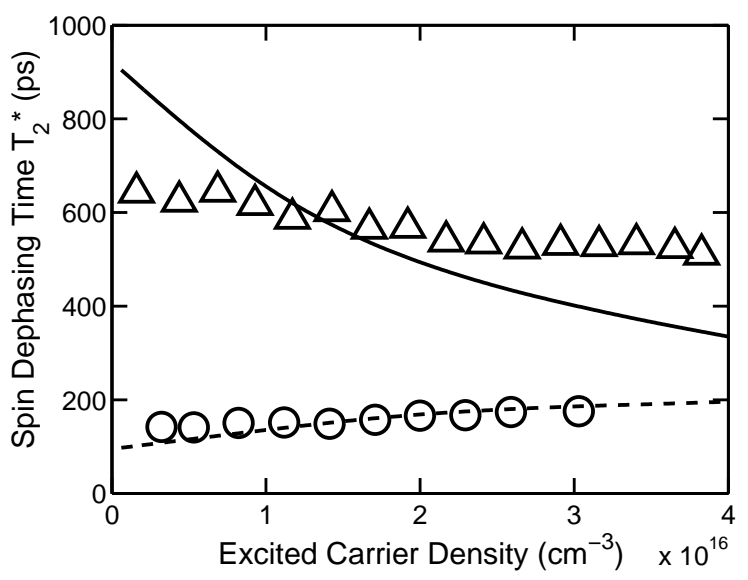

FIG. 2: Measured (symbols) and calculated (lines) spin dephasing times vs. photoexcited carrier density for doped (upper curves) and undoped (lower curves) bulk GaAs at $T=4 \mathrm{~K}$ and with $B=4 \mathrm{~T}$ external magnetic field. The doping density is $3.6 \times 10^{16} \mathrm{~cm}^{-3}$ (calculation) and $(3.6 \pm 0.3) \times 10^{16} \mathrm{~cm}^{-3}$ (experiment).

For these two scenarios, the interplay of the different scattering mechanisms (carrier-carrier, carrier-phonon, and carrier-impurity) and screening, and thus the influence of the optically excited carrier density on the spin-dephasing time are different.

Figure 2 already shows the pronounced influence of the doping density on the spin-dephasing time, and we investigate it now in more detail. The theoretical and experimental results in Fig. 3 are obtained by varying the doping density for a fixed density of optically excited carriers of $2 \times 10^{16} \mathrm{~cm}^{-3}$. We find good agreement of the measured data points with our microscopic calculation including only itinerant-electron dynamics, which suggests that the peak of the spindephasing time at intermediate doping densities can be explained without invoking a metal-insulator transition. ${ }^{7} \mathrm{~A}$ qualitative explanation of this peak is provided by the motional narrowing relation ${ }^{2,32}$

$$
\tau_{\text {spin }} \propto \frac{1}{\tau_{\mathrm{p}}} \quad \text { if }\langle\Omega\rangle \tau_{\mathrm{p}} \ll 1 .
$$

In Eq. (18), $\langle\Omega\rangle$ is the effective Larmor frequency due to the DP spin splitting averaged over the electrons taking part in the spin-precession and dephasing dynamics. The spin and momentum relaxation times are denoted by $\tau_{\text {spin }}$ and $\tau_{\mathrm{p}}$, respectively. For low and moderate doping densities, i.e., for densities up to $10^{17} \mathrm{~cm}^{-3}$ we are in the motional narrowing regime because the Larmor frequency $\boldsymbol{\Omega}(\boldsymbol{k})$ of the precession of an electron is proportional to $k^{3}$. Thus it becomes large only for electrons excited at larger momenta, which is the case at higher doping concentrations for optical excitation beyond the quasi Fermi-level of the relaxed electrons. The combination of elastic impurity scattering and mainly inelastic Coulomb scattering leads to a decrease of the effective momentum scattering time $\tau_{\mathrm{p}}$ with increasing doping density. Via the motional narrowing relation (18) this translates to a longer spin-dephasing time. For higher doping concentrations, the motional narrowing regime described by the inequality in Eq. (18) is left and the spin-dephasing time drops with increasing doping concentration. ${ }^{2}$

For a more quantitative understanding of the doping density dependence in Fig. 3, we analyze the contributions of the different scattering mechanisms. To this end, we also show in Fig. 3 the spin-dephasing time for the two artificial cases of (i) only elastic impurity scattering, and (ii) no impurity scattering. For case (ii) we still include carrier-carrier and carrier-phonon scattering, which lead to elastic and inelastic scattering events, but the inelastic processes have the larger available phase space and therefore dominate case (ii). For undoped GaAs, i.e., doping densities of less than $10^{15} \mathrm{~cm}^{-3}$, the full calculation is identical to case (ii), which shows that carrier-carrier and carrier-phonon scattering are responsible for the spin dephasing. ${ }^{35}$ For intermediate doping densities between $10^{15} \mathrm{~cm}^{-3}$ and $10^{17} \mathrm{~cm}^{-3}$ the rise of the spin-dephasing time towards a maximum at $8 \times 10^{16} \mathrm{~cm}^{-3}$ is determined by the interplay of the elastic impurity scattering, the predominantly inelastic carrier-carrier/phonon scattering, and the influence of screening on all three scattering processes. Inelastic or elastic scattering processes alone cannot explain the behavior of the spin-dephasing time in this region: If only elastic scattering is included, as in case (i), electrons are not scattered away from the higher $k$ states, in which they are excited by the laser; there they experience a faster precession, cf. Eq. (14), and thus dephase more quickly with increasing doping concentration. This effect counteracts the increasing effectiveness of the carrier-impurity scattering, which, in the picture of Eq. (18), leads to a shorter $\tau_{\mathrm{p}}$. If only carrier-carrier and carrierphonon scattering is included, as in case (ii), the optically excited carrier density of $2 \times 10^{16} \mathrm{~cm}^{-3}$ dominates over or 


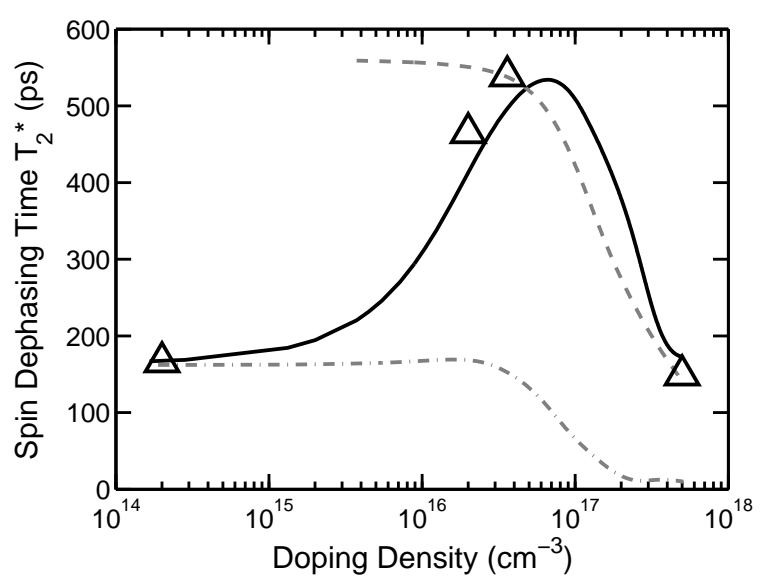

FIG. 3: Spin dephasing time vs. doping density at $4 \mathrm{~K}$ and with $4 \mathrm{~T}$ external magnetic field. Shown together with the calculated result including all scattering mechanisms (solid line) are curves obtained by including only impurity scattering (dashed grey line) and without impurity scattering (dash-dotted grey line). The triangles are experimental values. The excited carrier density for calculation and experiment is always $N_{\text {exc }}=2 \times 10^{16} \mathrm{~cm}^{-3}$.

is comparable to the doped carrier density for doping concentration of up to several $10^{16} \mathrm{~cm}^{-3}$, so that no pronounced density dependence is to be expected for these scattering mechanisms alone. ${ }^{36}$ For high doping concentrations beyond $10^{17} \mathrm{~cm}^{-3}$, the optically excited carriers cannot be scattered out of the high $k$ states in which they are created, because of the high density of relaxed electrons. Then, by Eq. (14), the precession of the excited electrons becomes too fast for the timescales of elastic [case(i)] scattering, inelastic scattering [case(ii)] or their combination [full result], so that the spin-dephasing time experiences a pronounced drop. ${ }^{2}$ The complex behavior of the doping-density dependence of the spin-dephasing time is thus quantitatively explained by the interplay of the different elastic and inelastic scattering mechanisms, and the influence of screening. This picture is essentially supported by a reanalysis of the data of Fig. 3 in Ref. 33. Last, but not least, we wish to stress that the numerical results provide a microscopic picture of motional narrowing in spin dephasing, i.e., an increasing $T_{2}^{*}$ with decreasing momentum scattering time, and the breakdown of this behavior for fast electronic precession. We do not need to resort to established analytical results for DP spin dephasing, ${ }^{23}$ which do not work over the whole density regime and for pronounced non-equilibrium conditions. ${ }^{34}$

\section{CONCLUSION}

We presented results for the spin dynamics of itinerant electrons due to the DP spin relaxation mechanism for a wide range of doping concentrations, and under elevated excitation conditions. Our calculations yield quantitative results for the DP spin relaxation process, which show the occurrence and breakdown of motional narrowing in spin dephasing for elevated optical excitation conditions. We find a maximum of the spin dephasing-time on a subnanosecond timescale at intermediate doping concentrations close to the density where, at low excitation conditions, a metal-insulator transition occurs. By numerically investigating the different contributions to the spin-dephasing time under elevated excitation conditions, we showed that the maximum of spin dephasing times in the intermediate doping regime is due to the interplay of elastic and inelastic scattering mechanisms, as well as screening.

\section{Acknowledgments}

A grant for CPU time from the NIC Jülich is gratefully acknowledged. We thank the Graduiertenkolleg 792 of the German Science Foundation (DFG) for financial support, and Ming-Wei Wu for helpful discussions.

* Present address: University of Konstanz and Center for Applied Photonics, 78457 Konstanz, Germany

$\dagger$ URL: http:\\www.physik.uni-kl.de\schneider 
1 D. D. Awschalom, D. Loss, and N. Samarth, eds., Semiconductor Spintronics and Quantum Computation (Springer, Berlin, 2002).

2 I. Žutić, J. Fabian, and S. Das Sarma, Rev. Mod. Phys. 76, 323 (2004).

3 J. M. Kikkawa and D. D. Awschalom, Phys. Rev. Lett. 80, 4313 (1998).

4 J. S. Sandhu, A. P. Heberle, J. J. Baumberg, and J. R. A. Cleaver, Phys. Rev. Lett. 86, 2150 (2001).

${ }^{5}$ G. M. Müller, M. Römer, D. Schuh, W. Wegscheider, J. Hübner, and M. Oestreich, Phys. Rev. Lett. 101, 206601 (2008).

${ }^{6}$ M. I. Dyakonov and V. I. Perel, Soviet Physics JETP 33, 1053 (1971).

7 R. I. Dzhioev, K. V. Kavokin, V. L. Korenev, M. V. Lazarev, B. Y. Meltser, M. N. Stepanova, B. P. Zakharchenya, D. Gammon, and D. S. Katzer, Phys. Rev. B 66, 245204 (2002).

8 M. Furis, D. L. Smith, S. A. Crooker, and J. L. Reno, Appl. Phys. Lett. 89, 102102 (2006).

9 M. Beck, C. Metzner, S. Malzer, and G. H. Döhler, Europhys. Lett. 75, 597 (2006).

${ }_{11}$ M. W. Wu and C. Z. Ning, Eur. Phys. J. B 18, 373 (2000).

${ }^{11}$ W. H. Lau, J. T. Olesberg, and M. E. Flatté, Phys. Rev. B 64, 161301(R) (2001).

12 D. Stich, J. Zhou, T. Korn, R. Schulz, D. Schuh, W. Wegscheider, M. W. Wu, and C. Schüller, Phys. Rev. Lett. 98, 176401 (2007).

13 J. H. Jiang and M. W. Wu, Phys. Rev. B 79, 125206 (2009).

14 A. Dyson and B. K. Ridley, Phys. Rev. B 69125211 (2004).

15 Z. G. Yu, S. Krishnamurthy, M. van Schilfgaarde, and N. Newman, Phys. Rev. B 71, 245312 (2005).

16 H. Haug and S. W. Koch, Quantum Theory of the Optical and Electronic Properties of Semiconductors (World Scientific, Singapore, 2004), 4th ed.

17 M. W. Wu and H. Metiu, Phys. Rev. B 61, 2945 (2000).

18 M. M. Glazov and E. L. Ivchenko, JETP 99, 1279 (2004).

19 C. Lechner and U. Rössler, Phys. Rev. B 72, 045311 (2005).

${ }^{20}$ W. Schäfer and M. Wegener, Semiconductor Optics and Transport Phenomena (Springer, Berlin, 2002).

21 R. Binder and S. W. Koch, Progress Quant. Electronics 19, 307 (1995).

22 F. Rossi and T. Kuhn, Rev. Mod. Phys. 74, 895 (2002).

${ }^{23}$ G. E. Pikus and A. N. Titkov, in Optical Orientation, edited by F. Meier and B. P. Zakharchenya (North-Holland, Amsterdam, 1984), pp. 73-131.

24 R. Winkler, Spin-Orbit Coupling Effects in Two-Dimensional Electron and Hole Systems (Springer, Berlin, 2003).

25 M. Z. Maialle, Phys. Rev. B 54, 1967 (1996).

26 H. C. Schneider, J. P. Wüstenberg, O. Andreyev, K. Hiebbner, L. Guo, J. Lange, L. Schreiber, B. Beschoten, M. Bauer, and M. Aeschlimann, Phys. Rev. B 73, 081302(R) (2006).

27 R. Bratschitsch, Z. Chen, S. T. Cundiff, E. A. Zhukov, D. R. Yakovlev, M. Bayer, G. Karczewski, T. Wojtowicz, and J. Kossut, Appl. Phys. Lett. 89, 221113 (2006).

28 Z. G. Chen, S. G. Carter, R. Bratschitsch, P. Dawson, and S. T. Cundiff, Nature Physics 3, 265 (2007).

29 D. J. Hilton and C. L. Tang, Phys. Rev. Lett. 89, 146601 (2002).

30 M. Krauß , M. Aeschlimann and H. C. Schneider, Phys. Rev. Lett. 100, 256601 (2008).

31 T. Östreich, K. Schönhammer, and L. J. Sham, Phys. Rev. Lett. 75, 2554 (1995).

32 M. A. Brand, A. Malinowski, O. Z. Karimov, P. A. Marsden, R. T. Harley, A. J. Shields, D. Sanvitto, D. A. Ritchie, and M. Y. Simmons, Phys. Rev. Lett. 89, 236601 (2002).

33 K. Shen, Chin. Phys. Lett 26, 067201 (2009).

34 P. H. Song and K. W. Kim, Phys. Rev. B 66, 035207 (2002).

${ }^{35}$ Curve (i) is not plotted for densities below $4 \times 10^{15} \mathrm{~cm}^{-3}$ because for low impurity concentrations there are hardly any impurity scattering events contributing to spin dephasing, so that the spin-dephasing time rapidly approaches a timescale of $10 \mathrm{~ns}$.

${ }^{36}$ For doping concentrations larger than $2 \times 10^{16} \mathrm{~cm}^{-3}$ the influence of screening keeps the carrier-carrier and carrier-phonon scattering alone from becoming more important for the spin dephasing. 